\title{
In Vitro and In Vivo Modeling of Hydroxypropyl Methylcellulose (HPMC) Matrix Tablet Erosion Under Fasting and Postprandial Status
}

\author{
Benjamin Guiastrennec ${ }^{\prime}$ - Erik Söderlind ${ }^{2,3}$ - Sara Richardson ${ }^{4}$ - Alexandra Peric ${ }^{5}$. \\ Martin Bergstrand '
}

Received: 21 October 2016 / Accepted: 24 January 2017 / Published online: 2 February 2017

(C) The Author(s) 2017. This article is published with open access at SpringerLink.com

\begin{abstract}
Purpose To develop a model linking in vitro and in vivo erosion of extended release tablets under fasting and postprandial status.

Methods A nonlinear mixed-effects model was developed from the in vitro erosion profiles of four hydroxypropyl methylcellulose (HPMC) matrix tablets studied under a range of experimental conditions. The model was used to predict in vivo erosion of the HPMC matrix tablets in different locations of the gastrointestinal tract, determined by magnetic marker monitoring. In each gastrointestinal segment the $\mathrm{pH}$ was set to physiological values and mechanical stress was estimated in USP2 apparatus rotation speed equivalent.
\end{abstract}

Results Erosion was best described by a Michaelis-Menten type model. The maximal HPMC release rate $\left(\mathrm{V}_{\mathrm{MAX}}\right)$ was affected by $\mathrm{pH}$, mechanical stress, HPMC and calcium hydrogen phosphate content. The amount of HPMC left at which the release rate is half of $\mathrm{V}_{\text {MAX }}$ depended on $\mathrm{pH}$ and calcium hydrogen phosphate. Mechanical stress was estimated for

Electronic supplementary material The online version of this article (doi: | 0. | 007/s | | 095-0 | 7-2 | |3-7) contains supplementary material, which is available to authorized users.

Martin Bergstrand

martin.bergstrand@farmbio.uu.se

Pharmacometrics Group, Department of Pharmaceutical Biosciences, Uppsala University, Box 591, 75124 Uppsala, Sweden

2 Pharmaceutical Technology and Development, AstraZeneca, Gothenburg, Sweden

3 Present address: Janssen Pharmaceutica NV, Turnhoutseweg 30, B-2340 Beerse, Belgium

4 Advanced Drug Delivery, Pharmaceutical Sciences, Innovative Medicines and Early Development, AstraZeneca, Gothenburg, Sweden

5 Drug Metabolism and Pharmacokinetics, Cardiovascular and Metabolic Diseases, Innovative Medicines and Early Development, AstraZeneca, Gothenburg, Sweden stomach (39.5 rpm), proximal (93.3 rpm) and distal (31.1 rpm) small intestine and colon (9.99 rpm).

Conclusions The in silico model accurately predicted the erosion profiles of HPMC matrix tablets under fasting and postprandial status and can be used to facilitate future development of extended release tablets.

KEY WORDS food effect · hydroxypropyl methylcellulose . in vitro in vivo correlation · magnetic marker monitoring

NONMEM

\section{ABBREVIATIONS}

$\mathrm{Y}$

$\eta$

$\mathrm{AIC}$

API

BOV

BSV

BTV

COV

$\mathrm{COV}_{50}$

$\mathrm{COV}_{\mathrm{MED}}$

$\mathrm{CV}$

DCP

ECov

$E_{D C P-K M}$

EDCP-VMAX

$E_{\text {MAX }}$

EMK4M-

VMAX

$E_{\mathrm{pH}-\mathrm{KM}}$

$\mathrm{E}_{\mathrm{pH}-\mathrm{VMAX}}$
Power factor parameter

BTV of $V_{\text {MAX }}$

Akaike information criterion

Active pharmaceutical ingredient

Between occasion variability

Between subject variability

Between tablet variability

A covariate

$\mathrm{COV}$ value leading to half of the maximal effect Median value of $\mathrm{COV}$

Coefficient of variation

Calcium hydrogen phosphate

Relative change in $\mathrm{TV}_{\mathrm{PRM}}$ for each unit change of $\mathrm{COV}$ in reference to $\mathrm{COV}_{\mathrm{MED}}$

Relative effect of DCP on the typical value of $\mathrm{K}_{\mathrm{M}}$

Relative effect of DCP on the typical value of $\mathrm{V}_{\text {MAX }}$

Maximal saturable effect of $\mathrm{COV}$ on $\mathrm{TV}_{\mathrm{PRM}}$ Relative effect of MK4M on the typical value of $\mathrm{V}_{\text {MAX }}$

Relative effect of $\mathrm{pH}$ on the typical value of $\mathrm{K}_{\mathrm{M}}$ Relative effect of $\mathrm{pH}$ on the typical value of $\mathrm{V}_{\text {MAX }}$ 


\begin{tabular}{|c|c|}
\hline ER & Extended release \\
\hline$E_{\text {rpm-VMAX }}$ & $\begin{array}{l}\text { Relative effect of mechanical stress on the typical } \\
\text { value of } V_{\text {MAX }}\end{array}$ \\
\hline FOCE-I & $\begin{array}{l}\text { First-order conditional estimation method with } \\
\text { interaction }\end{array}$ \\
\hline Gl & Gastrointestinal \\
\hline HPMC & Hydroxypropyl methylcellulose \\
\hline $\mathrm{HPMC}_{\text {dose }}$ & $\begin{array}{l}\text { Total amount of HPMC initially present in the } \\
\text { tablet }\end{array}$ \\
\hline HPMC tablet & $\begin{array}{l}\text { Total amount of HPMC remaining in the tablet } \\
\text { at a given time }\end{array}$ \\
\hline$K_{M}$ & $\begin{array}{l}\text { Amount of HPMC in tablet at which the release } \\
\text { rate is half of } V_{\text {MAX }}\end{array}$ \\
\hline MK4M & $\begin{array}{l}\text { Proportion of high molecular weight HPMC in } \\
\text { the tablet }\end{array}$ \\
\hline $\mathrm{MK}_{4} \mathrm{M}_{50-}$ & MK4M leading to a decrease of $50 \%$ in the \\
\hline VMAX & typical value of $V_{\text {MAX }}$ \\
\hline MMM & Magnetic marker monitoring \\
\hline OFV & Objective function value \\
\hline PRM & A model parameter \\
\hline PWR & Power coefficient parameter \\
\hline $\mathrm{R}$ & Basal HPMC release rate parameter \\
\hline RSE & Relative standard error \\
\hline SCM & Stepwise covariate modeling \\
\hline $\mathrm{S}_{\mathrm{COV}}$ & Effect slope of $\mathrm{COV}$ on $\mathrm{TV}_{\mathrm{PRM}}$ \\
\hline SITT & Small intestinal transit time \\
\hline$T V_{K M}$ & Typical value of the $\mathrm{K}_{\mathrm{M}}$ parameter \\
\hline$T_{\text {PRM }}$ & Typical value of a parameter \\
\hline TV VMAX & Typical value of the $V_{\text {MAx }}$ parameter \\
\hline USP2 & $\begin{array}{l}\text { United States pharmacopeia dissolution appa- } \\
\text { ratus } 2\end{array}$ \\
\hline$V_{\text {MAX }}$ & Maximal HPMC release rate \\
\hline VPC & Visual predictive checks \\
\hline
\end{tabular}

\section{INTRODUCTION}

Extended release (ER) dosage forms are widely used to optimize drug concentration in plasma and improve patient compliance. Their main application lies in the reduction of the dosing frequency for drugs with short elimination half-life and in the reduction of side effects occurring for drugs exhibiting highly variable plasma concentrations. A common ER dosage form is the hydrophilic matrix tablet where an active pharmaceutical ingredient (API) is mixed with a hydrophilic polymer. Hydroxypropyl methylcellulose (HPMC) is frequently used to create hydrophilic matrices due to a safe and biodegradable profile, the compatibility with numerous drugs and the high drug load capacity. Upon ingestion of an ER tablet, water will diffuse into its core, causing the HPMC matrix to swell and form a rubbery gel. Hydrophilic API are released by diffusion into the dissolution media, while less soluble API as well as the HPMC polymer itself are being released by progressive erosion of the gel layer (1-4). The molecular weight and proportion of HPMC within an ER tablet can be modified to optimize the release rate of an API to a specific therapeutic need.

In vitro dissolution testing is a cornerstone in the development of solid dosage forms. These tests are designed and used for product quality control, but also to predict in vivo erosion time profiles by identifying the influential factors (i.e. covariates) of drug release. In the case of HPMC matrix tablets numerous influential factors linked to the characteristics of the polymer (e.g. molecular weight, degree of substitution), the formulation and the API (e.g. solubility) have been reported $(3,5)$. In vitro testing experiments such as the United States pharmacopeia dissolution apparatus 2 (USP2) are typically conducted under a range of static experimental conditions (i.e. constant $\mathrm{pH}$ and mechanical stress) to characterize the effect of covariates on the release rate, but disregard the dynamic changes encountered in vivo along the gastrointestinal (GI) tract and between prandial statuses (6).

Magnetic marker monitoring (MMM) is a non-invasive, high resolution technique allowing the measurement in realtime of the erosion and the GI location of solid dosage forms. MMM relies on the measurement of the magnetic dipole generated by a magnetically labeled dosage form (7-9). More recently the MMM technique has been used to predict the in vivo GI transit of solid dosage form under different prandial statuses using in silico models (8).

The success of developing an ER tablet formulation with appropriate in vivo release characteristics relies on the ability to accurately quantify the effect of influential factors of the in vivo drug release, these factors include: tablet formulation, conditions throughout the GI tract and effect of prandial status. In silico models have been widely applied to in vitro predictions, yet only a few of these models have been utilized to make in vivo predictions of erosion time profiles (10-12). Hence, the present study aimed to link in vitro dissolution experiment data to in vivo erosion of ER tablets and to evaluate the effect of prandial status. For this purpose an in silico approach was used to 1) characterize the effect of formulation and experimental conditions based on in vitro erosion time profiles of four HPMC matrix tablets, 2) predict the in vivo erosion time profiles from a previously published MMM study under fasting and postprandial status (13).

\section{MATERIALS AND METHODS}

\section{Tablet Formulations}

Four ER hydrophilic matrix tablet formulations were tested (Table I). The formulations no. 1 to 3 contained a fixed amount of total HPMC $(40 \%)$ but variable proportions of high (303 kDa, Methocel K4M Premium; DowWolf, USA) 
Table I Hydrophilic Matrix Tablets' Formulation ( 13)

\begin{tabular}{|c|c|c|c|c|}
\hline \multirow[t]{2}{*}{ Component } & \multicolumn{4}{|c|}{ Formulation } \\
\hline & No. I & No. 2 & No. 3 & No. 4 \\
\hline Methocel K4M \% (w/W) & 23.0 & 10.0 & - & - \\
\hline Methocel KIOOLV \% (w/W) & 17.0 & 30.0 & 40.0 & 20.0 \\
\hline Calcium hydrogen phosphate \% (w/w) & 57.6 & 57.6 & 57.6 & 77.6 \\
\hline Black iron oxide \% (w/w) & 1.4 & 1.4 & 1.4 & 1.4 \\
\hline Sodium stearyl fumarate $\%(w / W)$ & 1.0 & 1.0 & 1.0 & 1.0 \\
\hline
\end{tabular}

and low (128 kDa, Methocel K100LV Premium; DowWolf, USA) molecular weight HPMC. Formulation no. 4 contained a reduced amount of HPMC $(20 \%)$ and only used the low molecular weight HPMC. Calcium hydrogen phosphate (DCP) (DI-TAB®; Innophos, USA) was used as filler in all formulations to reach a total weight of about $350 \mathrm{mg}$. All tablets also contained fixed amounts of sodium stearyl fumarate (1\%) (Pruv®; Moehs, Spain) and black iron oxide (1.4\%) (Sicovit Schwarz 80E172, BASF), the latter being used as a label for the MMM.

\section{In Vitro Experiments}

The erosion properties of each formulation were evaluated in vitro using an USP2 (Hanson SR8 Plus Dissolution Test Station, USA) equipped with a stationary basket (6). The erosion was investigated under a range of experimental conditions: mechanical stress (i.e. rotation speed), $\mathrm{pH}$ and ionic strength (Table II). Biorelevant media were selected to mimic gastric ( $\mathrm{pH} 1.2$ and $232 \mathrm{mOsm} / \mathrm{kg}$ ionic strength) and small intestine (pH 6.8 and $98 \mathrm{mOsm} / \mathrm{kg}$ ionic strength) fluids. A third medium (pH 6.8 and $232 \mathrm{mOsm} / \mathrm{kg}$ ionic strength) was selected to differentiate the effects of $\mathrm{pH}$ and ionic strength on the release of HPMC. Each experimental setting was carried out as triplicate to ensure the reproducibility of the assay. In total, the in vitro data included 24 previously-published and 45 newly-generated tablet erosion profiles (Table II) (13).

Throughout this analysis HPMC release was used as surrogate for tablet erosion (Fig. 1). The concentration of HPMC in the release medium was determined by size exclusion chromatography with dual multi-angle light scattering and refractive index detection (SEG-MALS/RI) (13). A model published by fain et al. was used to correlate the in vitro release of HPMC from the tablet to the decrease of the magnetic moment which the MMM method relies upon (13).

\section{Clinical Study}

In vivo tablet erosion data were obtained from five healthy, young (range 27-34 years) male volunteers involved in a single center, open-label study (13). The study was conducted over six occasions separated by wash-out periods of at least $24 \mathrm{~h}$ following the last MMM measurement. Formulations no. 1 and 2 were administered under fasting status and formulations no. 3 and 4 under both fasting and postprandial status. Magnetized tablets were administered along with $240 \mathrm{~mL}$ of water at room temperature after an overnight fast of at least $10 \mathrm{~h}$ (fasting status) or $30 \mathrm{~min}$ after ingestion of a standardized high fat meal (postprandial status). MMM measurements of 10 min were initiated at the time of ingestion and conducted at intervals of $30 \mathrm{~min}$ to monitor tablet location in the GI tract and quantify the erosion. The GI tablet location was reported as three distinct regions: stomach, small intestine and colon. Volunteers were given standardized meal-break for lunch $(+4 \mathrm{~h})$, afternoon snack $(+6 \mathrm{~h})$ and dinner $(+10 \mathrm{~h})$. During these breaks no MMM measurements were conducted and volunteers were encouraged to walk or sit. Fluid intakes were also standardized from the time of tablet ingestion and up to $10 \mathrm{~h}$ afterward. MMM measurements were carried out until the net magnetization of the tablet was below $15 \%$ of its initial value ( $\sim 60 \%$ HPMC released) but no longer than $14 \mathrm{~h}$ post administration.
Table II Summary of In Vitro USP2 Apparatus Experimental Conditions

\begin{tabular}{|c|c|c|c|c|}
\hline & \multicolumn{2}{|l|}{$\mathrm{pH} 1.2$} & \multicolumn{2}{|l|}{$\mathrm{pH} 6.8$} \\
\hline & Formulation & $\begin{array}{l}\text { Mechanical stress } \\
\text { (rpm) }\end{array}$ & Formulation & $\begin{array}{l}\text { Mechanical stress } \\
\text { (rpm) }\end{array}$ \\
\hline \multirow[t]{4}{*}{ Low ionic strength (98 mOsm/kg) } & no. I & - & no. I & $50^{a} / 100 / 150$ \\
\hline & no. 2 & - & no. 2 & $25 / 50^{\mathrm{a}}$ \\
\hline & no. 3 & - & no. 3 & $25 / 50^{a} / 100 / 150$ \\
\hline & no. 4 & - & no. 4 & $50^{a} / 100$ \\
\hline \multirow[t]{4}{*}{ High ionic strength (232 mOsm/kg) } & no. 1 & $25 / 50^{a} / 150$ & no. I & 50 \\
\hline & no. 2 & $50^{a} / 100$ & no. 2 & 50 \\
\hline & no. 3 & $25 / 50^{\mathrm{a}} / 150$ & no. 3 & 50 \\
\hline & no. 4 & $50^{\mathrm{a}}$ & no. 4 & - \\
\hline
\end{tabular}

${ }^{\text {a }}$ Experiments part of a previous publication (I3) 


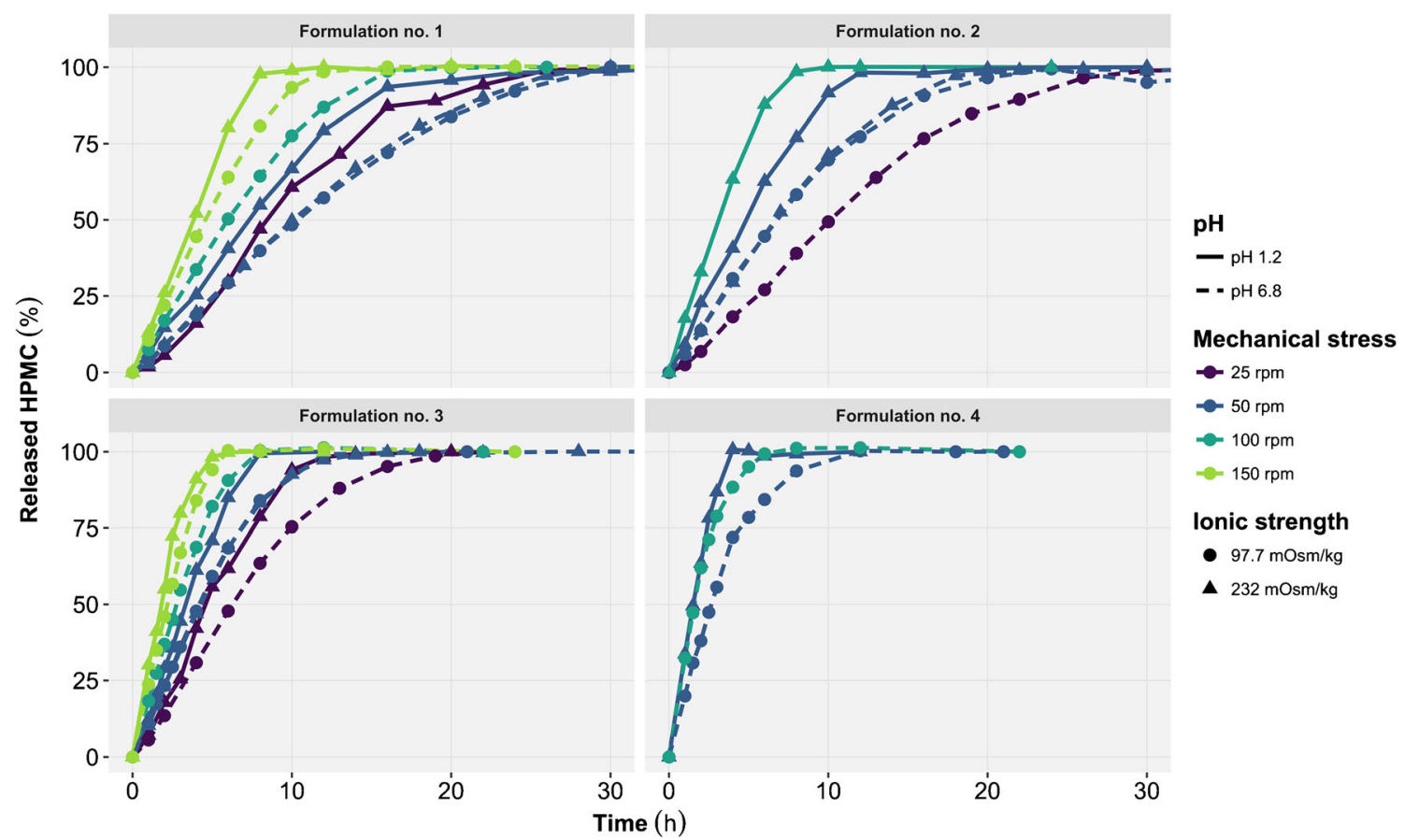

Fig. I Released hydroxypropyl methylcellulose (HPMC) time profiles generated by the in vitro USP2 apparatus experiments. The profiles are represented as function of formulation (panel), $\mathrm{pH}$ (line type), mechanical stress (color) and ionic strength (symbol). Each point represents the mean of triplicate experiments.

All volunteers signed a written informed consent form. The study was performed in accordance with the ethical standards of the 1964 Helsinki declaration and its later amendments and was approved by the ethics committee of the medical chamber of Thüringen (Ethikkommission der Landesärztekammer Thüringen).

\section{Model Building}

\section{Overview of the Analysis}

The present analysis has been conducted in three sequential steps. First, an in silico model was developed using the in vitro HPMC release time profiles to characterize the effect of influential factors (i.e. tablet formulation, experimental conditions). Secondly, the in silico model was applied to the prediction of in vivo HPMC release time profiles by using GI location (MMM data) to dynamically mimic the tablet environment properties throughout the GI. Lastly, the in silico model was used to simulate the in vivo HPMC release time profile for different formulation and prandial status combinations.

\section{Software}

The data analysis was performed using a nonlinear mixed-effects modeling approach implemented in the NONMEM software (v.7.3) and aided by functionalities of the PsN toolkit (v.4.4.2) (14). The first-order conditional estimation method with interaction (FOCE-I) and the ADVAN13 subroutine were used for parameter estimation. Graphical analysis and data management were conducted in $\mathrm{R}$ (v.3.2.0).

\section{Model Selection and Diagnostics}

Model selection was based on differences in the objective function value (OFV) using a significance level of $p<0.05$ for nested models and on a comparison of the Akaike information criterion (AIC) for non-nested models. Graphical diagnostics, scientific plausibility and parameter uncertainty were also considered in the model evaluation. Parameter uncertainty reported as relative standard error (RSE) was obtained from the NONMEM sandwich estimator. Values missing at random, due to poor sample quality in the in vitro data $(17 \%)$, were excluded from the analysis. No missing data were reported in the in vivo dataset.

Visual predictive checks (VPC) were used throughout model development to evaluate the predictive performance of the in vitro and in vivo models. This evaluation is done graphically by comparing the 5th, 50th and 95th percentiles of the observed data to the $95 \%$ confidence interval around the 5 th, 50th and 95th percentiles of the simulated $(n=1000)$ data, grouped together within bins of the independent variable (i.e. time) (15). Prediction-corrected VPG were used to evaluate model performance across all experimental conditions and 
formulations within a single graphic. With predictioncorrected VPC the observed and simulated values of the dependent variable (i.e. released HPMC) are for each bin normalized to the typical model prediction (i.e. no parameter variability) at the median of the independent variable in the bin (15).

As per study protocol, no additional MMM measurements were conducted once the net magnetization of the ER tablet was below $15 \%$ of its initial value. To properly evaluate model performance, VPC with censoring were used in the diagnostic of the in vivo model by mimicking the study protocol. Censored VPG allowed the simulation of HPMC release profiles to be interrupted when the stopping criteria (i.e. low magnetization of the tablet) was met $(15,16)$.

\section{In vitro tablet erosion model}

The in silico tablet erosion model was developed to characterize the in vitro HPMC release profiles. The predictive performance of a published mechanism-based model describing the HPMC release rate as function of the tablet surface area (Eq. 1) (10) was compared to a Michaelis-Menten type model (Eq. 2).

$$
\frac{d H P M C_{\text {tablet }}}{d t}=-R \cdot\left(\frac{H P M C_{\text {dose }}}{\text { Tabletweight }}\right) \cdot\left(\frac{\text { Tablet weight } \cdot H P M C_{\text {tablet }}}{H P M C_{\text {dose }}}\right)^{\gamma}
$$

where $\mathrm{HPMC}_{\text {dose }}$ is the total amount of HPMC initially present in the tablet, $\mathrm{HPMC}_{\text {tablet }}$ the total amount of HPMC remaining in the tablet at a given time, $\mathrm{R}$ the basal HPMC release rate, and $\gamma$ the power factor.

$\frac{d H P M C_{\text {tablet }}}{d t}=-\frac{V_{M A X} \cdot H P M C_{\text {tablet }}}{K_{M}+H P M C_{\text {tablet }}}$

where $\mathrm{V}_{\text {MAX }}$ is the maximal HPMC release rate and $\mathrm{K}_{\mathrm{M}}$ corresponds to $\mathrm{HPMC}_{\text {tablet }}$ for which the release rate is half of $\mathrm{V}_{\text {MAX }}$. Both candidate models were pre-selected for their potential ability to describe the changes in the tablet erosion rate as a function of $\mathrm{HPMC}_{\text {tablet }}$ - the erosion rate starting at a high and fairly constant value before gradually decreasing over time (Fig. 1). The models were implemented using differential equations to facilitate the inclusion of time varying covariates effect (e.g. increase in $\mathrm{pH}$ following gastric emptying) on the release rate of HPMC.

Addition of a lag-time to the HPMC release rate was evaluated to account for the presence of potential onset of HPMC release which may arise from the initial tablet hydration (17). The effect of experimental conditions (mechanical stress, $\mathrm{pH}$ and ionic strength) and tablet formulations (proportion of high molecular weight HPMC and DCP) were tested on the structural model parameters using a stepwise covariate modeling (SCM) approach as implemented in the PsN toolkit (14). In the forward inclusion step of the SCM, each covariate was tested in a univariate manner on each model parameter-including $\mathrm{R}$ and $\gamma$ for the mechanism-based model and $\mathrm{V}_{\text {MAX }}$ and $\mathrm{K}_{\mathrm{M}}$ for the Michaelis-Menten model. The most significant covariate-parameter relationship was then included in the model and taken forward. This process was performed in an iterative manner using the model from the previous step as starting point and testing the remaining covariate-parameter relationship until no more significant $(p<0.05)$ relationship was identified. In the backward deletion step of the SCM, the included covariates were removed one by one to more strictly $(p \geq 0.01)$ evaluate the statistical significance and omit potential redundant covariate-parameter relationship. All covariates were first tested by the SCM using relative linear effects as illustrated in Eq. 3:

$P R M=T V_{P R M} \cdot\left(I+S_{C O V} \cdot\left(C O V-C O V_{M E D}\right)\right)$

where $\mathrm{TV}_{\mathrm{PRM}}$ is the estimated typical value of the model parameter (PRM), $\mathrm{S}_{\mathrm{COV}}$ is effect slope of the covariate (COV) on $\mathrm{TV}_{\mathrm{PRM}}$ and $\mathrm{COV}$ MED is the median value of COV. The effect of mechanical stress and of the amount of high molecular weight HPMC were tested in vitro under a wider range of values enabling the evaluation of nonlinearity in the covariate-parameter relationship (Table II). The non-linear relationships were evaluated using power (Eq. 4) and saturable Michaelis-Menten (Eq. 5) covariate-parameter relationships upon the linear covariate inclusion by the SCM.

$P R M=T V_{P R M} \cdot\left(\frac{C O V}{C O V_{M E D}}\right)^{P W R}$
$P R M=T V_{P R M} \cdot\left(1+\frac{E_{M A X} \cdot C O V}{C O V_{50}+C O V}\right)$

where PWR is the power coefficient, $\mathrm{E}_{\mathrm{MAX}}$ the maximal saturable effect of $\mathrm{COV}$ (bound between 0 and -1 in the case of an inhibitory effect) on the TV $\mathrm{PRM}_{\text {of }} \mathrm{PRM}$ and $\mathrm{COV}_{50}$ the $\mathrm{COV}$ value leading half of $\mathrm{E}_{\mathrm{MAX}}$. The between tablet variability (BTV) was investigated on model parameters and was assumed to follow a lognormal distribution. Proportional, additive and combined models were evaluated to describe the residual unexplained variability (i.e. measurement error, model misspecifications).

\section{In Viwo Tablet Erosion Model}

The selected in vitro model was used as a starting point to predict the in vivo HPMC release profiles data. Several modifications were carried out on the in vitro model to allow for proper description of in vivo data. 


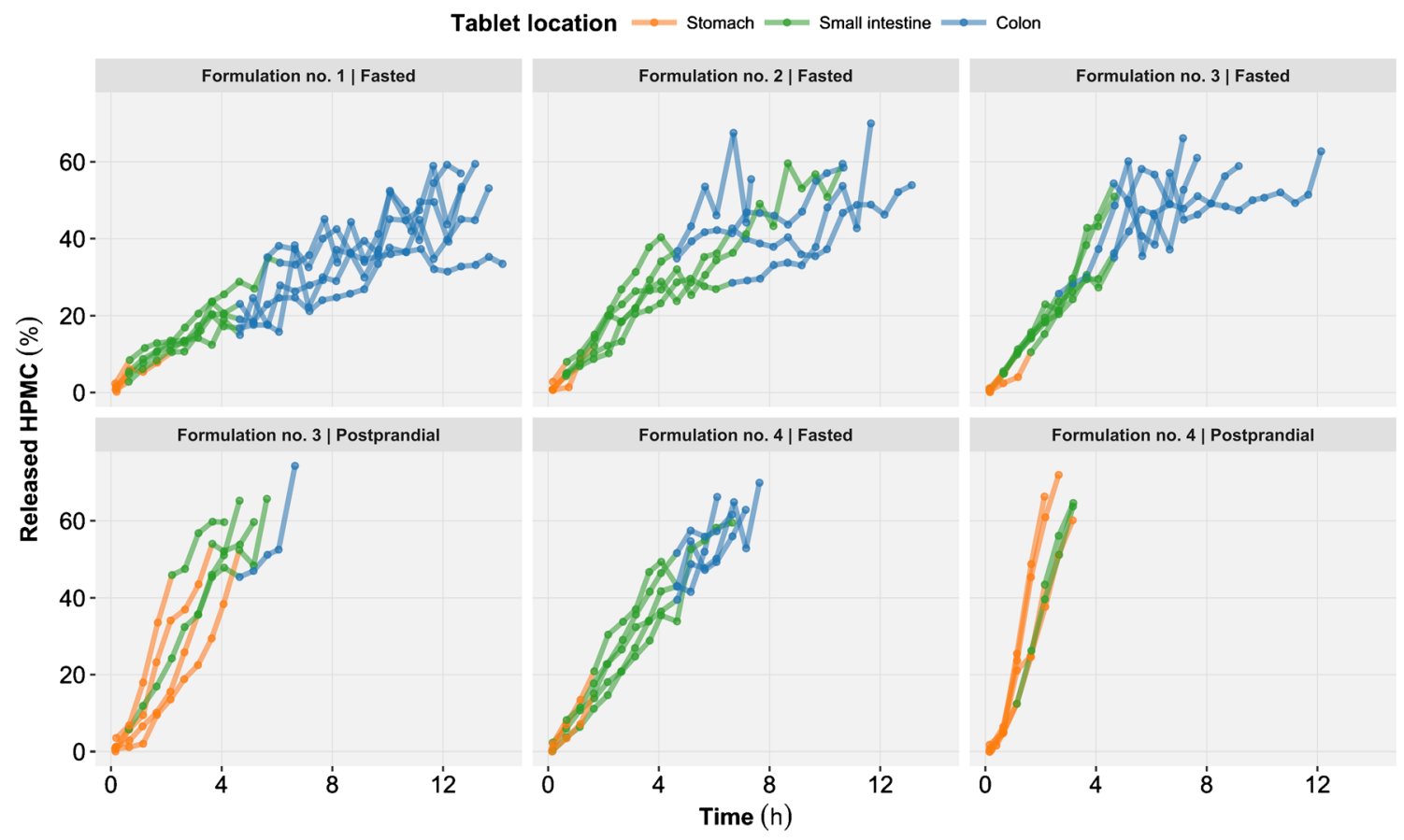

Fig. 2 Released hydroxypropyl methylcellulose (HPMC) time profiles generated by the in vivo clinical study. The profiles are represented as function of formulation and prandial status (panel) as well as the tablet location in the gastrointestinal tract (color).

First, the typical values of all structural model parameters and the covariate effects were fixed to the in vitro model estimates (i.e. not re-estimated). Secondly, the variability in the model parameters was modified to account for the different layers of variability of the in vivo data, namely: the between subject variability (BSV) and between occasions variability (BOV) arising from the crossover design[34]. Thirdly, the residual unexplained variability structure was re-evaluated to account for the measurement error of the MMM. Fourthly, the GI tract tablet location (i.e. stomach, small intestine and colon) obtained from the MMM data was used to dynamically adjust the local $\mathrm{pH}$ and mechanical stress in the model. Graphical exploration of the data revealed consistent changes across individuals in the HPMC release rate throughout the small intestine (Fig. 2). To provide the model with the flexibility to account for these changes, three alternative approaches were tested to segment the small intestine into its proximal and distal parts. In the first approach, the individual small intestinal transit time (SITT) from the data along with the fractional length of proximal $(45 \%)$ and distal $(55 \%)$ small intestine were used to compute the time of transfer between the two segments (18). In the second approach the time of transfer between the proximal and distal small intestine for the population was estimated on relation to the time of gastric emptying. In the third approach it was fixed to one hour after the gastric emptying, as reported by Bergstrand et al. in a similar study (10). The resulting GI location (i.e. stomach, proximal small intestine, distal small intestine and colon) was used in the model to dynamically adjust the properties of the tablet environment. Literature values (mean and standard deviation) obtained from a similar population (Table III) were used as prior information to generate a distribution of $\mathrm{pH}$ within each GI location. The extent of mechanical stress on the tablet was estimated and expressed as USP2 apparatus rotation speed unit equivalent (rpm). Finally, for formulation no. 3 and 4, a postprandial effect was implemented by adjusting the distribution of $\mathrm{pH}$ values in the stomach, while the postprandial effect in other GI segments was assumed to be negligible (Table III). A potential postprandial effect was also investigated on the stomach mechanical stress.

Table III pH Value in the Different Gastrointestinal Segments as a Function of the Prandial Status $[35,36]$

\begin{tabular}{llll}
\hline Gastro-intestinal segment & Prandial status & pH value & \\
\cline { 3 - 4 } & & Mean & SD \\
\hline Stomach & Fasting $^{\text {a }}$ & 1.73 & 0.52 \\
& Postprandial $^{a}$ & 4.90 & 0.81 \\
Proximal small intestine & - & 6.63 & 0.53 \\
Distal small intestine & - & 7.49 & 0.46 \\
Colon & - & 6.63 & 0.67 \\
\hline
\end{tabular}

a Mean and standard deviation (SD) calculated from the median and interquartile range according to a method described by Wan et al. [37] 

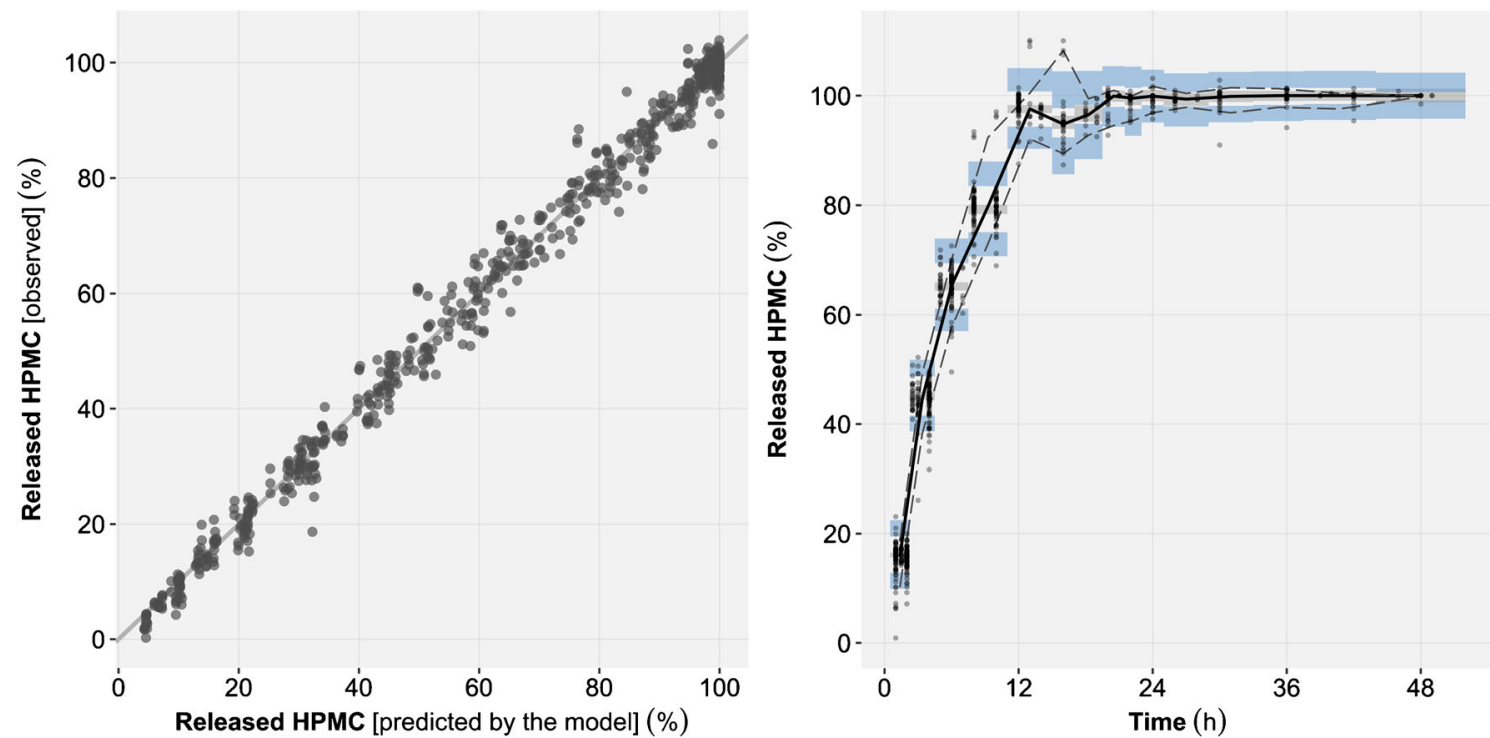

Fig. 3 Left: goodness-of-fit plot of the in vitro model showing the correlation between the observed and the typical model predictions of released hydroxypropyl methylcellulose (HPMC) for all formulations and experimental conditions. Right: Prediction corrected visual predictive checks (VPC) of in vitro released HPMC time course for all formulations and experimental conditions. With prediction corrected VPC the observed and simulated values of the dependent variable (released HPMC) are for each bin normalized to the typical model prediction (i.e. no between tablet variability) at the median of the independent variable (time) in the bin. The median (bold line), 5th and 95th percentiles (dashed lines) of the observed data are compared to the 95\% confidence intervals (shaded areas) for the median (grey), the 5 th and 95 th percentiles of the simulated $(n=1000)$ data (blue).

\section{Simulations}

Model simulations were performed to illustrate the expected in vivo HPMC release time profile with regard to formulation and prandial status. In order to generate a high number of new MMM GI location profiles, a first set of simulations were run from a previously published Markov model describing the GI transit of solid dosage form in fasting and postprandial status (8). The HPMC release profiles were then simulated by using the newly generated GI location profiles in the in vivo tablet erosion model. Finally, the simulations were summarized for each combination of formulation and prandial status by graphically representing the median and the $90 \%$ prediction interval of the simulated data.

\section{RESULTS}

\section{In Vitro Tablet Erosion Model}

The Michaelis-Menten model (Eq. 2; AIC = 1959) displayed superior predictive performance than the mechanism-based model (Eq. 1; AIC = 2118). Moreover, the MichaelisMenten model appropriately described the HPMC release rate of all four formulations under all studied experimental conditions and was thus selected as in vitro model (Fig. 3, Supplementary Materials S1).

The parameter estimates for the selected in vitro HPMC release model are reported in Table IV. Estimation of a lag-time $(0.179 \mathrm{~h})$ to account for the initial tablet hydration resulted in a significant model fit improvement. The BTV was estimated to a coefficient of variation $(\mathrm{CV})$ of $5.96 \%$ for $\mathrm{V}_{\mathrm{MAX}}$, but was no longer supported on $\mathrm{K}_{\mathrm{M}}$

Table IV Parameter Estimates for the In Vitro Tablet Erosion Model

\begin{tabular}{|c|c|}
\hline Parameter (units) & Estimate (RSE\%) \\
\hline$V_{\operatorname{MAX}}\left(m g_{H P M d} / h\right)$ & $22.0(2.5)$ \\
\hline $\mathrm{K}_{M}\left(m g_{H P M C}\right)$ & $34.2(6.1)$ \\
\hline Lag-time $(h)$ & $0.179(15)$ \\
\hline$M^{\prime} 4 M_{50-V M A X}(\% w / W)$ & | $8.7(3.5)$ \\
\hline$S_{D C P-K M}\left(\% w / w^{-1}\right)^{a}$ & $0.0522(33)$ \\
\hline$S_{\text {DCP-VMAX }}\left(\% w / w^{-1}\right)^{a}$ & $0.0332(24)$ \\
\hline $\mathrm{S}_{\mathrm{pH}-\mathrm{KM}}\left(\mathrm{pH} \text { unit }^{-1}\right)^{a}$ & $0.0935(15)$ \\
\hline $\mathrm{S}_{\mathrm{pH}-\mathrm{VMAX}}\left(\mathrm{pH} \text { unit }^{-1}\right)^{a}$ & $-0.0319(24)$ \\
\hline$S_{r p m-V M A X}\left(r p m^{-1}\right)^{a}$ & $0.0115(2.9)$ \\
\hline BTV $\vee_{\operatorname{MAX}}(C \vee \%)$ & $5.96(17)$ \\
\hline Additive error ${ }^{b}$ (mg $\left.g_{H P M C}\right)$ & $1.95(\mid 4)$ \\
\hline
\end{tabular}

BTV between tablet variability, CV coefficient of variation, DCP calcium hydrogen phosphate, $K_{M}$ hydroxypropyl methylcellulose (HPMC) amount left in tablet at which $\mathrm{V}_{\text {MAX }}$ is reduced by $50 \%$, MK4M 50 -VMAX amount of high molecular weight HPMC at which $\mathrm{V}_{\text {MAX }}$ is reduced by $50 \%$, RSE relative standard error, $S_{D C P-K M}$ DCP effect slope on $K_{M}, S_{D C P-V M A X}$ DCP effect slope on $V_{M A X}, S_{P H-K M} p H$ effect slope on $K_{M}, S_{p H-V M A X} p H$ effect slope on $V_{M A X}$, $S_{\text {rpm-VMAX }}$ mechanical stress effect slope on $V_{\text {MAX }}, V_{\text {MAX }}$ maximal HPMC release rate from the tablet

${ }^{a}$ covariate effects reported as relative change in the model parameter typical value for each unit change of the covariate in reference to its median

${ }^{\mathrm{b}}$ reported on the standard deviation scale 
upon inclusion of the covariate effects and therefore not retained in the selected model. The SCM identified significant effects of $\mathrm{pH}$, mechanical stress, the proportions of high molecular weight HPMC and DCP in the tablet on $\mathrm{V}_{\mathrm{MAX}}$ (Eq. 6) and of $\mathrm{pH}$ was well of the proportion of DCP in the tablet on $\mathrm{K}_{\mathrm{M}}$ (Eq. 7).

$V_{M A X}=T V_{V M A X} \cdot E_{r p m-V M A X} \cdot E_{p H-V M A X} \cdot E_{D C P-V M A X} \cdot E_{M K 4 M-V M A X} \cdot e^{\eta}$

$\kappa_{M}=T V_{K M} \cdot E_{p H-K M} \cdot E_{D C P-K M}$

where $\mathrm{TV}_{\mathrm{VMAX}}$ and $\mathrm{TV}_{\mathrm{KM}}$ represent the typical (i.e. formulation no. 3, pH 6.8, mechanical stress $=50 \mathrm{rpm}) \mathrm{pa}-$ rameter values of $\mathrm{V}_{\mathrm{MAX}}$ and $\mathrm{K}_{\mathrm{M}}$. $\mathrm{E}_{\text {rpm-VMAX }}, \mathrm{E}_{\mathrm{pH} \text {-VMAX, }}$ $\mathrm{E}_{\mathrm{DCP}-\mathrm{VMAX}}$ and $\mathrm{E}_{\mathrm{MK} 4 \mathrm{M}-\mathrm{VMAX}}$ represent the relative effects of mechanical stress, $\mathrm{pH}, \mathrm{DCP}$ and high molecular weight $\mathrm{HPMC}$, respectively, on $\mathrm{TV}_{\mathrm{VMAX}}$. The symbol $\eta$ represents the BTV on $\mathrm{V}_{\mathrm{MAX}}$. $\mathrm{E}_{\mathrm{pH}-\mathrm{KM}}$ and $\mathrm{E}_{\mathrm{DCP}-\mathrm{KM}}$ represent the relative effects of $\mathrm{pH}$ and $\mathrm{DCP}$ on $\mathrm{K}_{\mathrm{M}}$. $\mathrm{E}_{\mathrm{MK} 4 \mathrm{M} \text { - }}$ VMAX was best described by a saturable relationship (Eq. 8) which resulted in a model fit improvement over a linear relationship.

$E_{M K 4 M-V M A X}=1-\frac{M K 4 M}{M K 4 M_{50-V M A X}+M K 4 M}$

where MK4M is the proportion of high molecular weight HPMC and MK4M 50 -VMAX the MK4M leading to a decrease by half of $\mathrm{E}_{\mathrm{MK} 4 \mathrm{M}-\mathrm{VMAX}}$. In Eq. $8 \mathrm{E}_{\mathrm{MAX}}$ was not statistically different from -1 and hence was fixed to a full inhibitory effect in the model. No effect of the ionic strength could be detected on any of the model parameters. A schematic representation of the structural and covariate models is illustrated in Fig. 4. Moreover, a visual representation of the effect of significant covariate relationships on the released HPMC time profiles is provided in Fig. 5. Finally, the residual unexplained variability was best described by an additive error model.

\section{In Vivo Tablet Erosion Model}

Predictions from the selected in vivo tablet erosion model were overall in good agreement with the observed released HPMC time profiles (Fig. 6). Under the fasting status, HPMC release from the formulation no. 4 (i.e. lowest HPMC content) were systematically over predicted by the model (Fig. 7). Thus, the data from formulation no. 4 under fasting status were excluded during the final parameters estimation, but were included for model diagnostics and simulations. This approach was selected to prevent the introduction of an estimation bias in the model parameters due to model misspecification. The parameter estimates for the selected in vivo tablet erosion model

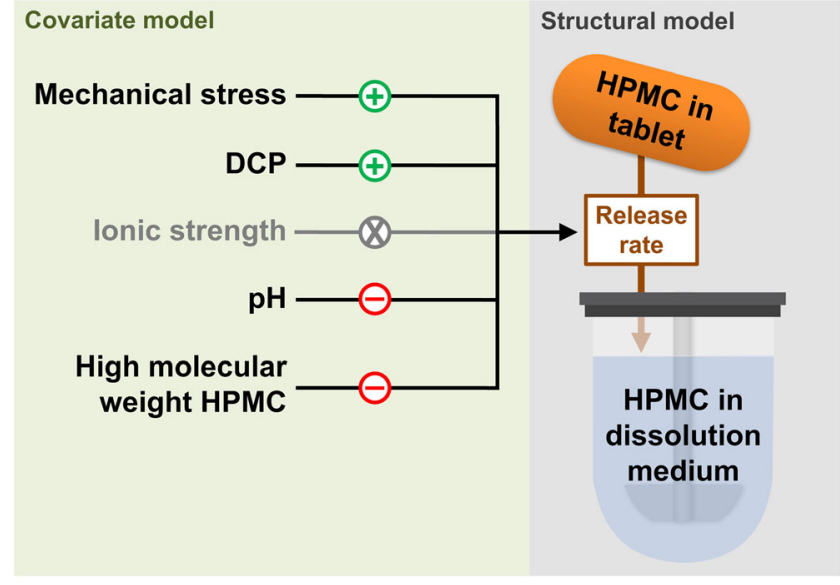

Fig. 4 Schematic representation of the structural model (right) where hydroxypropyl methylcellulose (HPMC) is being released from the tablet into the dissolution medium. The effects of covariate (left) on the tablet erosion rate are also represented. The green $(+)$ symbols represent a stimulation, the red $(-)$ symbols an inhibition and the grey $(x)$ symbol an absence of observable covariate effect on the tablet erosion rate. DCP: calcium hydrogen phosphate.

have been reported in Table $\mathrm{V}$ and the model code in the Supplementary Materials S2. The small intestine segmentation into its proximal and distal parts significantly improved model predictions. A model where the time of transfer between proximal and distal small intestine was fixed to one hour after gastric emptying gave the best results. The mechanical stress was estimated for stomach (39.5 rpm), proximal small intestine $(93.3 \mathrm{rpm})$, distal small intestine $(31.1 \mathrm{rpm})$ and colon $(9.99 \mathrm{rpm})$. Reestimation of the lag-time $(0.410 \mathrm{~h})$ significantly improved model predictions. The estimated BSV and BOV on $\mathrm{V}_{\mathrm{MAX}}$ were 14.9 and $15.5 \%$ respectively. BSV was also tested on $\mathrm{K}_{\mathrm{M}}$ but was not statistically significant and thus was not retained in the selected in vivo model. A postprandial effect was implemented on stomach $\mathrm{pH}$, although the estimation of an additional effect on mechanical stress was not supported by the data. The residual unexplained variability was best described by a combined (i.e. additive and proportional) model.

\section{DISCUSSION}

The present study demonstrated how the data from in vitro dissolution experiment could be integrated via an in silico approach to accurately predict in viwo tablet erosion of ER tablets under fasting and postprandial conditions. The in silico model was developed using in vitro USP2 apparatus dissolution data and applied to the characterization of tablet formulations and experimental conditions effects on the HPMC matrix tablet erosion. The model was then used to the predict the in vivo tablet erosion profiles by integrating MMM tablet location 


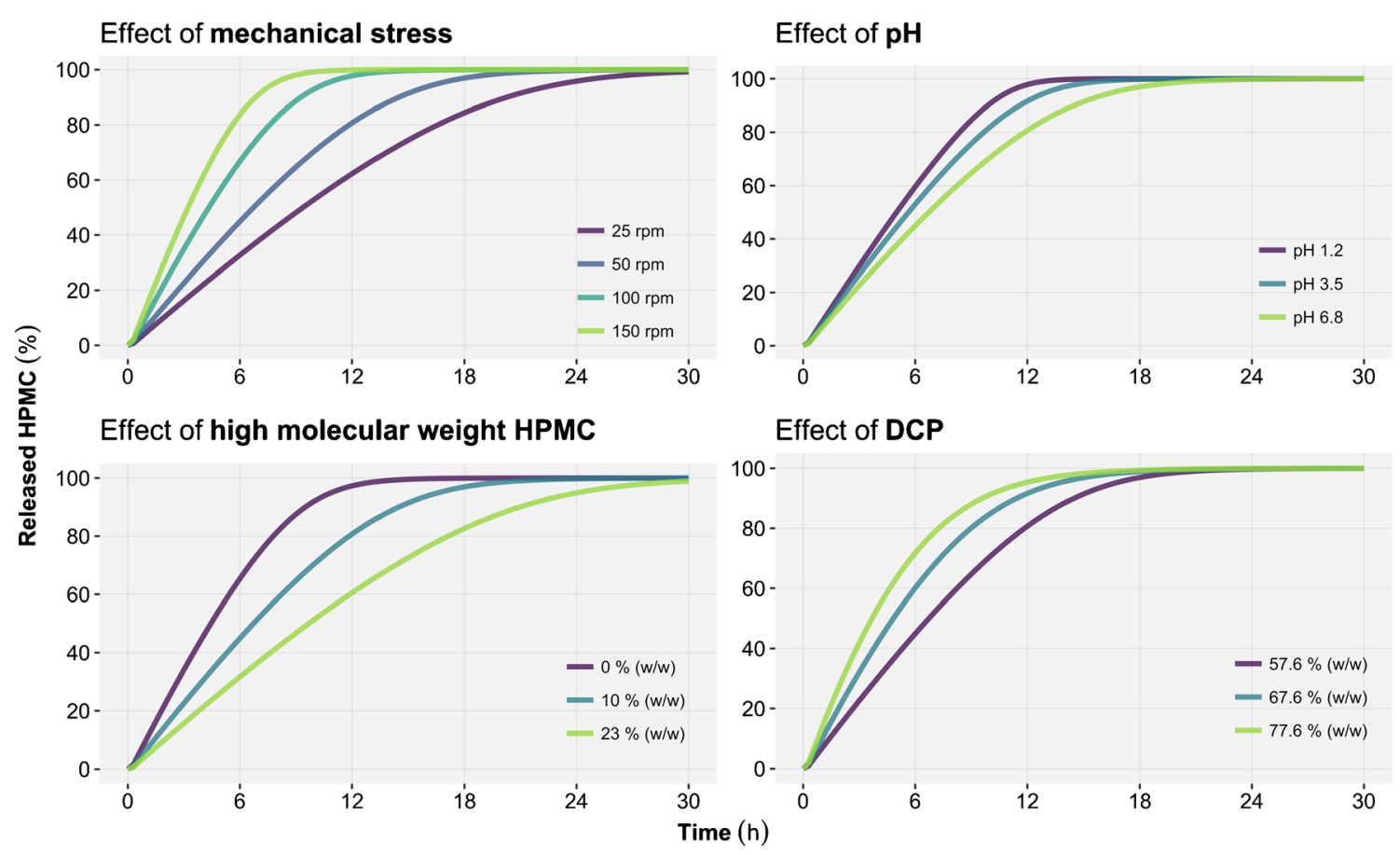

Fig. 5 Illustration of the predicted in vitro effect of: mechanical stress (top left), $\mathrm{pH}$ (top right), high molecular weight hydroxypropyl methylcellulose (HPMC) (bottom left) and calcium hydrogen phosphate (DCP) (bottom right) on released HPMC time profiles.

data. The resulting in silico model could be applied to the prediction of the in viwo erosion for similar hydrophilic matrix formulations and to the refinement of the experimental conditions used in vitro.

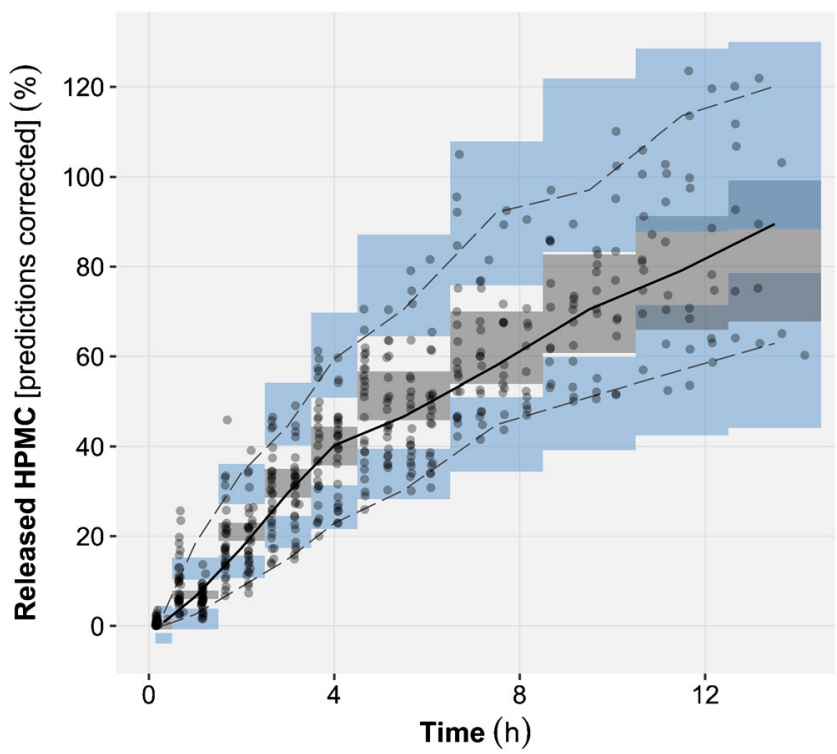

Fig. 6 Prediction-corrected visual predictive check of the in vivo released hydroxypropyl methylcellulose (HPMC) time profiles for all formulations and prandial statuses. With prediction corrected VPC the observed and simulated values of the dependent variable (released HPMC) are for each bin normalized to the typical model prediction (i.e. no between tablet variability) at the median of the independent variable (time) in the bin. The median (bold line), 5th and 95th percentiles (dashed lines) of the observed data are compared to the $95 \%$ confidence intervals (shaded areas) for the median (grey), the 5th and 95th percentiles of the simulated $(n=1000)$ data (blue).
Multiple in vitro tablet erosion models with various levels of complexity are available in the literature (21-23). These models were not investigated in the present study since they could not easily be applied to the dynamic conditions (e.g. changes in $\mathrm{pH}$ and mechanical stress) encountered in vivo throughout the GI tract. The in vitro predictive performance of the mechanism-based model developed by Bergstrand et al. in a similar study (10) was compared to a Michaelis-Menten model. Both models appropriately predicted the in vitro erosion time profiles, however the Michaelis-Menten model $(\mathrm{AIC}=1959)$ gave a better description of the data than the mechanism-based model (AIC $=2118)$. The mechanism based model assumes that the shape of a tablet remains constant throughout its disintegration, interestingly this assumption may have not been fully supported by the data.

The estimation of a lag-time of erosion $(0.179 \mathrm{~h})$ to describe the initial tablet hydration significantly improved the in vitro model predictions $(17,24)$. A $1 \mathrm{~h}$ lag-time has also been reported in vitro by Tajarobi et al. with similar tablet formulation (24). Unlike the present study the published value was not estimated but obtained from a direct observation at the first time point of the experiment which is likely to explain the difference between them. The estimation of a more pronounced in vivo lag-time $(0.410 \mathrm{~h})$ is also in line with values reported by Ghimire et al. and is believed to arise from the higher viscosity of the fluids in the stomach (17).

The identified effects of tablet formulations and experimental conditions on the release rate of HPMC were all in line with reported covariates of HPMC matrix tablet erosion $(1,3,17,24)$. The ratio of heavy to low molecular weight 


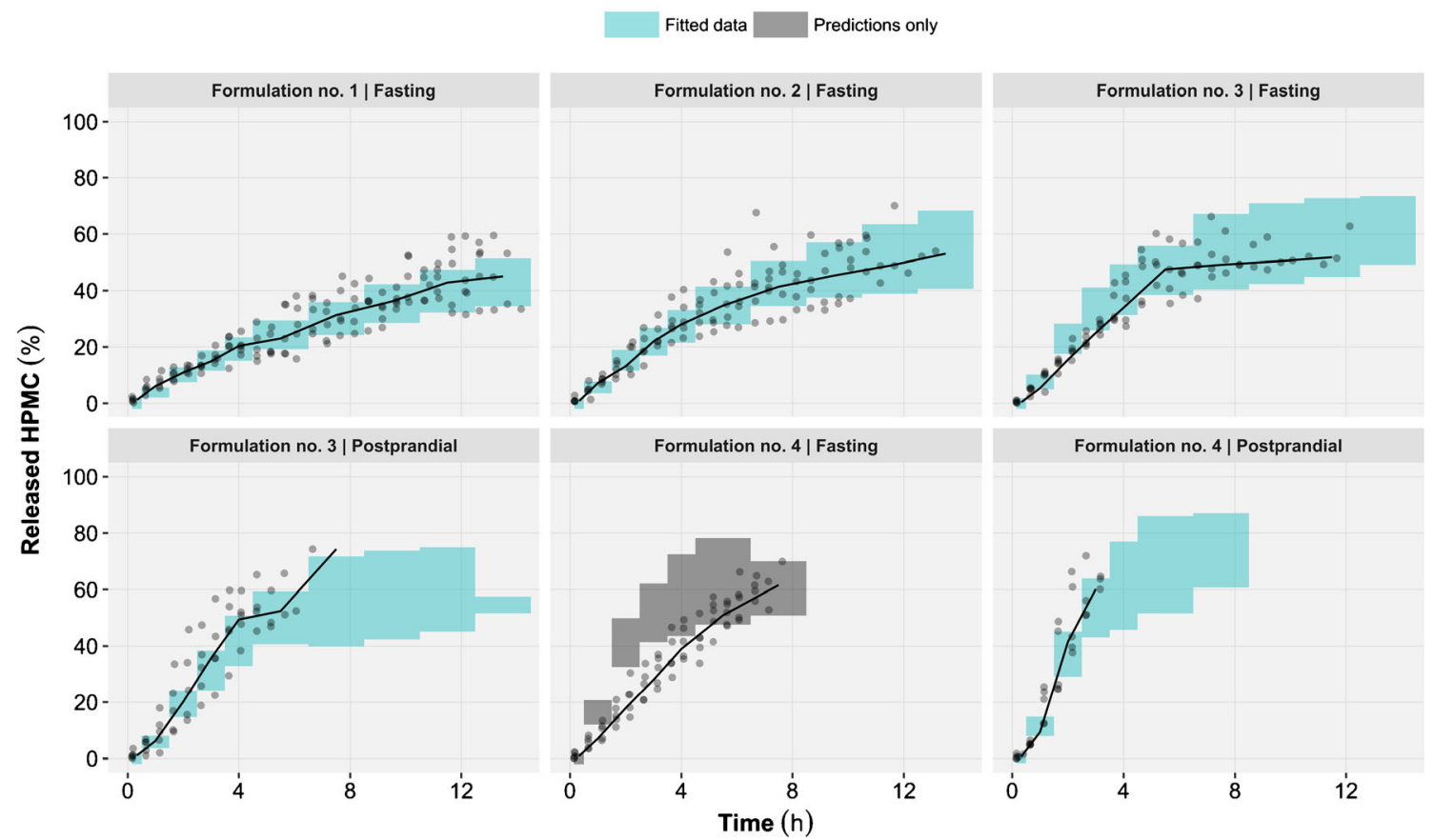

Fig. 7 Visual predictive checks of the in vivo released hydroxypropyl methylcellulose (HPMC) time profiles, stratified by formulation and prandial status. The simulated released HPMC were censored once the net magnetization of a tablet was below I $5 \%$ of its initial value. Censoring was utilized in the simulations as per study protocol where no subsequent measurements were performed once a threshold was met thus allowing to properly diagnose the model predictions. For each panel, the median (black lines) of the observed data (grey dots) is compared to the $95 \%$ confidence interval of the simulated $(n=1000)$ median (shaded area). The panels in blue represent the data used during the model development process, while the panel in grey represents the data where only predictions were made.

HPMC (formulation no. 1 to 3) was predicted to be inversely and non-linearly correlated to $\mathrm{V}_{\mathrm{MAX}}$. Reduced water diffusion rate into the tablet has been reported with higher HPMC molecular weight $(3,13,25)$. An increased fraction of DCP (formulation no. 4) was predicted to increase both $\mathrm{V}_{\mathrm{MAX}}$ and $\mathrm{K}_{\mathrm{M}}$. Previous studies have reported that formulation additives such as DCP or mannitol increase the diffusion of water

Table V Parameter Estimates for the In Vivo Tablet Erosion Model

\begin{tabular}{|c|c|}
\hline Parameter (units) & Estimate (RSE\%) \\
\hline $\mathrm{RPM}_{\mathrm{ST}}(r p m)$ & $39.5(24)$ \\
\hline $\mathrm{RPM}_{\mathrm{PSI}}(r p m)$ & $93.3(12)$ \\
\hline $\mathrm{RPM}_{\mathrm{DSI}}(r p m)$ & $3||.(2 \mid)$ \\
\hline $\mathrm{RPM}_{\mathrm{CO}}(r p m)$ & $9.99(54)$ \\
\hline Lag-time $(h)$ & $0.410(9.6)$ \\
\hline BSV on $V_{\operatorname{MAX}}(C V \%)$ & $14.9(23)$ \\
\hline BOV on $V_{\operatorname{MAX}}(C V \%)$ & I $5.5(36)$ \\
\hline Proportional error (CV\%) & $14.2(13)$ \\
\hline Additive error ${ }^{\mathrm{a}}\left(\mathrm{mg}_{\text {HPMC }}\right)$ & $1.86(21)$ \\
\hline
\end{tabular}

BOV between occasion variability, BSV between subject variability, $C V$ coefficient of variation, HPMC hydroxypropyl methylcellulose, RPM $\mathrm{CO}$ mechanical stress in colon, $R P M_{D S /}$ mechanical stress in distal small intestine, $R P M_{P S I}$ mechanical stress in proximal small intestine, RPM $M_{S T}$ mechanical stress in stomach, RSE relative standard error

a reported on the standard deviation scale into the tablet and thus the maximal erosion rate as compared to a pure HPMC matrix tablets $(1,24)$. The predicted effect of $\mathrm{pH}$ on $\mathrm{V}_{\mathrm{MAX}}$ and $\mathrm{K}_{\mathrm{M}}$ is likely related to the presence of DCP, which solubility is altered by $\mathrm{pH}$ and hence affects the water entry into the tablet as described above (1). Mechanical stress had the strongest effect on $\mathrm{V}_{\mathrm{MAX}}$ and displayed a linear relationship across the tested range (25-150 rpm). This effect depicts individual polymer chains being disjointed from the gel matrix under the action of the shear forces (24). Unlike findings from Bergstrand et al., no significant effect of ionic strength was detected despite in vitro experiments conducted at 98 and $232 \mathrm{mOsm} / \mathrm{kg}$ with a $\mathrm{pH}$ of 6.8 (Table II) (10). The graphical overlay between the erosion profiles at low and high ionic strength (Fig. 1, formulations no. 1 and 2, mechanical stress $=$ $50 \mathrm{rpm}, \mathrm{pH}$ 6.8) indicates that this finding is related to the in vitro experiment data rather than model misspecifications. Others have reported the influence of the HPMC quality and of the filling agent's solubility on the sensibility of tablet erosion to ionic strength which could explain the differences with findings from Bergstrand et al. (26-28). Indeed, the formulations studied herein were mostly composed of poorly soluble DCP and used K-quality HPMC whereas, the formulations studied by Bergstrand et al. used a highly soluble API along with Equality HPMC (10).

The in vivo model predictions were overall in good agreement with the data. However, the predictions for formulation no. 4 under fasting status were unexpectedly poor (Fig. 7). 
This data was thus subsequently excluded from the final parameters estimation to the prevent introduction of a bias in the parameter estimates. Tablets with a HPMC content inferior to the percolation threshold $(\sim 30-35 \% w / w)$ such as formulation no. $4(20 \% w / w)$ are however likely to exhibit poor in vivo behavior. With an HPMC content below the percolation threshold the formed gel layer becomes weaker and more prone to disruption by the shear forces $(1,17)$. The predicted in vivo release of HPMC for formulation no. 4 (Fig. 8) should however be interpreted carefully.

Assuming homogenous $\mathrm{pH}$ and mechanical stress throughout the entire small intestine transit did not provide an optimal description of the data. Surprisingly, among the three tested small intestine segmentation approaches, the use of individual SITT did not perform well as opposed to a segmentation one hour post gastric emptying for all individuals. This finding could be due to a long tablet residence time in the ileocecal junction, whereas the transit time throughout the proximal small intestine might have been relatively constant (29).

The in vivo mechanical stress estimates were consistently lower in all GI segments than previously reported by Bergstrand et al. in a similar study with tablets containing an API (10). These discrepancies can potentially be explained by differences in the tablet formulation, in the present study tablets no API was used. In the in vivo model described by Bergstrand et al. significant effects of the ionic strength of the media and of the amount of API in the tablet were identified on the erosion rate. In addition, Bergstrand et al. tested two different values of intestinal ionic strength in the model which resulted in marginally different estimates of mechanical stress (10). As previously mentioned, the sensitivity of a formulation to ionic strength is function of the osmotic pressure generated by API and filling agent $(24,26)$. In the case of DCP, the generated osmotic pressure is limited due to its low solubility (24). This may explain the absence of an ionic strength effect in the studied formulations and consequently the discrepancies with the previously reported mechanical stress estimates (10).

The parameter estimates for the mechanical stress in the proximal small intestine was estimated to $93.3 \mathrm{rpm}$ before dropping to $31.1 \mathrm{rpm}$ in the distal small intestine. The estimate for proximal small intestine was more than two times the estimate for the stomach (39.5 rpm). The high proximal intestine estimate could be explained by some disruption or damage of the tablet or the gel layer throughout the gastric emptying process $(23,30)$. Additionally, while the estimate of mechanical stress in stomach mainly arose from the postprandial data due to the prolonged gastric residence, the estimates for small intestine and colon mainly arose from the fasting data. Hence, in the postprandial state tablets may be have been surrounded by meal components creating a viscous medium with low water content. Such effect of viscosity on the tablet erosion rate have been reported in vivo when tablets were ingested along with viscous meals $(31,32)$. The same
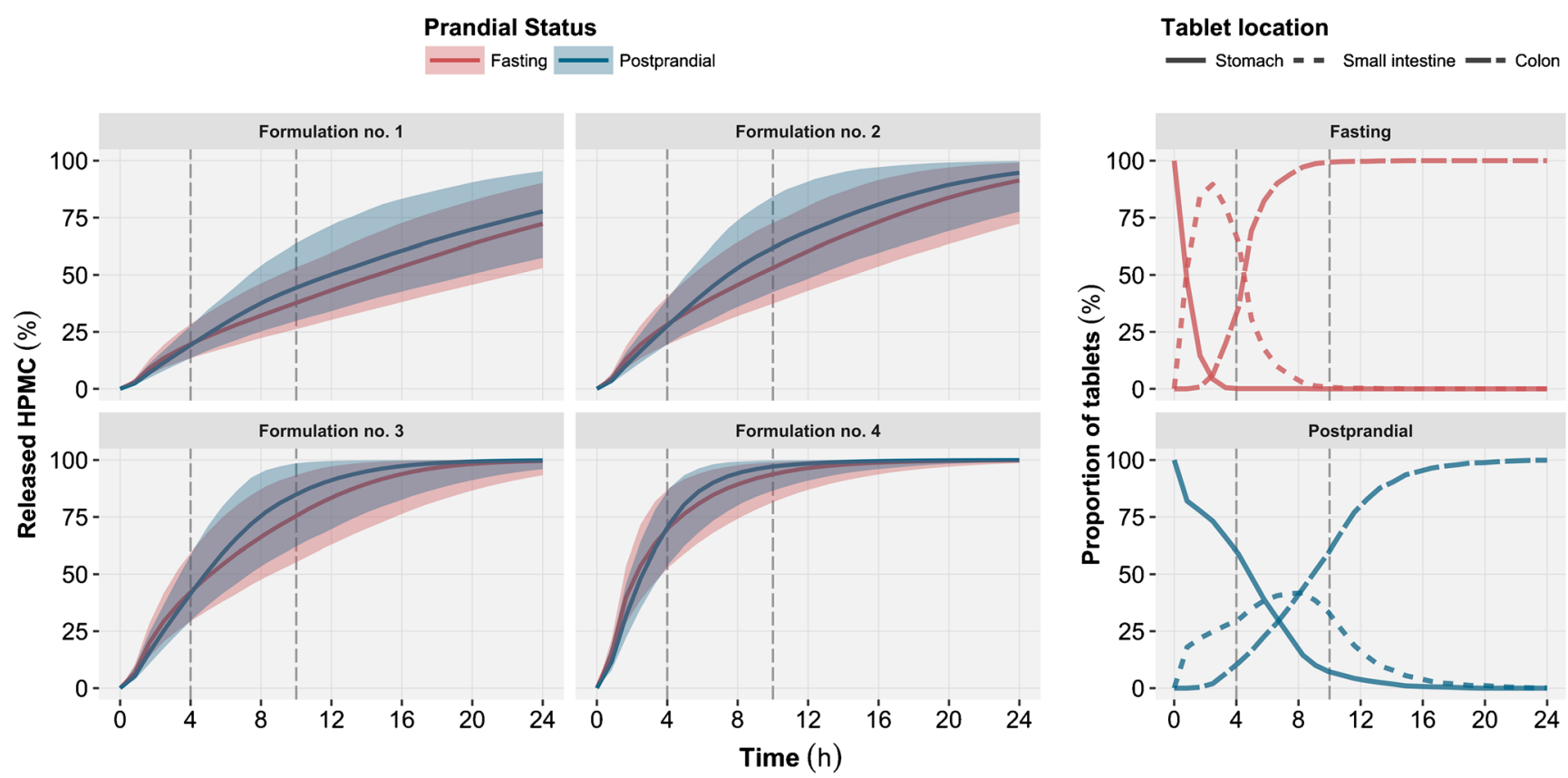

Fig. 8 Left: Simulated in vivo released hydroxypropyl methylcellulose (HPMC) time profiles (left) as a function of prandial status (color) and formulation (panel). The vertical dashed lines represent the time of lunch $(+4 \mathrm{~h})$ and dinner $(+10 \mathrm{~h})$ intake for both the fasting and postprandial conditions. For each simulated prandial status and formulation, the median (continuous line) and the $90 \%$ prediction interval (shaded area) were computed from I 000 simulated released HPMC profiles. Right: Graphical representation of the proportion of simulated tablet location in each gastrointestinal segment (line type) as function of the prandial status (color). These tablet locations were used by the in vivo model to generate the released HPMC profiles. 
mechanism may also explain the very small estimate of the mechanical stress in colon (9.99 rpm) as water is extensively being reabsorbed in that part of the intestine, which gradually increases the viscosity of the surrounding medium.

In the present study, the prandial effect was implemented as a change in stomach $\mathrm{pH}$, although in line with similar studies, no effect was observed on the mechanical stress $(10,33)$. While the authors do not exclude the hypothesis of an increased mechanical stress in postprandial stomach this effect was not supported by the current data where the tablet erosion data was limited in fasting stomach due to a short residence time (Fig. 2). Despite this, the model properly described the differences between fasting and postprandial status for formulation no 3. (Fig. 7) through the increased residence time in stomach and the relatively high mechanical stress encountered in stomach as compared to the mechanical stress encountered in distal small intestine and colon.

The developed in silico model was used to perform simulations of different formulation and prandial status (Fig. 8). The model also offers the opportunity to simulate new scenarios (e.g. new formulations) while accounting for the effect of covariates and the between subject variability in GI transit and $\mathrm{pH}$. To perform these simulations the in silico model was used along with a previously developed continuous Markov model describing the GI transit of solid dosage form under fasting and postprandial status (8). This implementation enables the simulation of new clinical trial design, which could be used to explore the HPMC release under different food patterns or with multiple tablet administration. Such simulations can be used to support of the development process by providing information on the expected typical tablet erosion profiles and the magnitude of variability. If used along with pharmacokinetic data, the model could be extended to predict the variability in the absorption profile of API released from ER formulations (19). It should be noted that the predictive performance of the proposed in viwo HPMC matrix tablet erosion model is appropriate for interpolation but has not been evaluated outside the studied range of covariates.

The present study is primarily devoted to ER tablets with an erosion-controlled drug release mechanism. The absence of API in the investigated tablets provided a fundamental understanding of the mechanisms of HPMC tablet erosion and the impact of covariates. Combined with API properties, this knowledge could enable to better predict in vivo behavior of API containing formulations. The drawback of this approach is that the in vivo absorption process of the studied formulations could not be evaluated. Furthermore, the proposed model relies on simplification of the shear forces and $\mathrm{pH}$ encountered in vivo. The MMM data used to determine the GI location of the tablets only reported three different locations, namely: stomach, small intestine and colon. In a similar study by Bergrstrand et al. more accurate MMM measurement allowed the distinction between proximal and distal stomach and the different sections of the colon which could have improved the current model predictions (10). The model also assumed a time-constant $\mathrm{pH}$ in the postprandial stomach, reports have however shown a return toward fasting values after several hours (20). Development of model describing the postprandial evolution of $\mathrm{pH}$ in stomach using data from frequent in vivo sampling could help to improve the current model predictions (20). The poor predictive performance of the model for formulation no. 4 under fasting status were unexpected. While it has been reported that tablets containing low amount of polymer are more likely to exhibit poor in vivo behavior, this may indicate that some factors in the in vitro-in vivo translation are yet to be characterized.

\section{CONCLUSION}

This work illustrates how an in silico erosion model for HPMC matrix tablets was developed using a simple set of in vitro USP2 apparatus dissolution profiles to predict the in vivo erosion. The presented methodology described how the in vitro effects of tablet formulations and experimental conditions were integrated in the in silico model to predict erosion time profiles of multiple HPMC matrix tablets under fasting and postprandial status. This study also exemplified how the in silico model can be used to inform decision-making through simulation of a typical tablet erosion profile and the extent of its variability.

\section{ACKNOWLEDGMENTS AND DISCLOSURES}

All studies described in the manuscript were sponsored by AstraZeneca. This work was contributed to the Orbito project (http://www.orbitoproject.eu) as side ground. Benjamin Guiastrennec and Martin Bergstrand have received research funding from AstraZeneca. Alexandra Peric, Sara Richardson are employees of AstraZeneca. Erik Söderlind was employee of AstraZeneca at the time of the study.

Open Access This article is distributed under the terms of the Creative Commons Attribution 4.0 International License (http://creativecommons.org/licenses/by/4.0/), which permits unrestricted use, distribution, and reproduction in any medium, provided you give appropriate credit to the original author(s) and the source, provide a link to the Creative Commons license, and indicate if changes were made.

\section{REFERENCES}

1. Tajarobi F, Abrahmsén-Alami S, Hansen M, Larsson A. The impact of dose and solubility of additives on the release from HPMC matrix tablets-identifying critical conditions. Pharm Res. 2009;26(6):1496-503. 
2. Viridén A, Abrahmsén-Alami S, Wittgren B, Larsson A. Release of theophylline and carbamazepine from matrix tabletsConsequences of HPMC chemical heterogeneity. Eur J Pharm Biopharm. 2011;78(3):470-9.

3. Maderuelo C, Zarzuelo A, Lanao JM. Critical factors in the release of drugs from sustained release hydrophilic matrices. J Control Release Elsevier BV. 2011;154(1):2-19.

4. Gajdošová M, Pěček D, Sarvašová N, Grof Z, Štěpánek F. Effect of hydrophobic inclusions on polymer swelling kinetics studied by magnetic resonance imaging. Int J Pharm. 2016;500(1-2):136-43.

5. Viridén A, Wittgren B, Andersson T, Larsson A. The effect of chemical heterogeneity of HPMC on polymer release from matrix tablets. Eur J Pharm Sci. 2009;36(4-5):392-400.

6. McAllister M. Dynamic dissolution: a step closer to predictive dissolution testing? Mol Pharm. 2010;7(5):1374-87.

7. Weitschies W, Blume H, Mönnikes H. Magnetic marker monitoring: high resolution real-time tracking of oral solid dosage forms in the gastrointestinal tract. Eur J Pharm Biopharm Elsevier BV. 2010;74(1):93-101.

8. Hénin E, Bergstrand M, Weitschies W, Karlsson MO. Metaanalysis of magnetic marker monitoring data to characterize the movement of single unit dosage forms though the gastrointestinal tract under fed and fasting conditions. Pharm Res. 2016;33(3):75162.

9. Weitschies W, Wilson CG. In vivo imaging of drug delivery systems in the gastrointestinal tract. Int J Pharm Elsevier BV. 201 1;417(12):216-26.

10. Bergstrand M, Söderlind E, Eriksson UG, Weitschies W, Karlsson MO. A semi-mechanistic modeling strategy to link in vitro and in vivo drug release for modified release formulations. Pharm Res. 2012;29(3):695-706.

11. Mujtaba A, Kohli K. In vitro / in vivo evaluation of HPMC / alginate based extended-release matrix tablets of cefpodoxime proxetil. Int J Biol Macromol Elsevier BV. 2016;89:434-41.

12. Otsuka K, Wagner C, Selen A, Dressman J. Prediction of in-vivo pharmacokinetic profile for immediate and modified release oral dosage forms of furosemide using an in-vitro-in-silico-in-vivo approach. J Pharm Pharmacol. 2015;67(5):651-65.

13. Jain AK, Söderlind E, Viridén A, Schug B, Abrahamsson B, Knopke C, et al. The influence of hydroxypropyl methylcellulose (HPMC) molecular weight, concentration and effect of food on in vivo erosion behavior of HPMC matrix tablets. J Control Release Elsevier BV. 2014;187:50-8.

14. Keizer RJ, Karlsson MO, Hooker A. Modeling and simulation workbench for NONMEM: tutorial on Pirana, PsN, and Xpose. CPT Pharmacometrics Syst Pharmacol. 2013;2:e50.

15. Bergstrand M, Hooker AC, Wallin JE, Karlsson MO. Predictioncorrected visual predictive checks for diagnosing nonlinear mixedeffects models. AAPS J. 2011;13(2):143-51.

16. Bergstrand M, Karlsson MO. Handling data below the limit of quantification in mixed effect models. AAPS J. 2009;1 1(2):371-80.

17. Ghimire M, Hodges LA, Band J, O'Mahony B, McInnes FJ, Mullen $\mathrm{AB}$, et al. In-vitro and in-vivo erosion profiles of hydroxypropylmethylcellulose (HPMC) matrix tablets. J Control Release Elsevier BV. 2010;147(1):70-5.

18. Valentin J. Basic anatomical and physiological data for use in radiological protection: reference values. Ann ICRP. 2002;32(3-4):1277.

19. Bergstrand M, Söderlind E, Eriksson UG, Weitschies W, Karlsson MO. A semi-mechanistic modeling strategy for characterization of regional absorption properties and prospective prediction of plasma concentrations following administration of new modified release formulations. Pharm Res. 2012;29(2):574-84.

20. Koziolek M, Schneider F, Grimm M, Mode C, Seekamp A, Roustom $\mathrm{T}$, et al. Intragastric $\mathrm{pH}$ and pressure profiles after intake of the high-caloric, high-fat meal as used for food effect studies. J Control Release. 2015;220:7 1-8.

21. Costa P, Sousa Lobo JM. Modeling and comparison of dissolution profiles. Eur J Pharm Sci. 2001;13(2):123-33.

22. Kaunisto E, Tajarobi F, Abrahmsen-Alami S, Larsson A, Nilsson B, Axelsson A. Mechanistic modelling of drug release from a polymer matrix using magnetic resonance microimaging. Eur J Pharm Sci. 2013;48(4-5):698-708.

23. Abrahamsson B, Pal A, Sjöberg M, Carlsson M, Laurell E, Brasseur JG. A novel in vitro and numerical analysis of shear-induced drug release from extended-release tablets in the fed stomach. Pharm Res. 2005;22(8):1215-26.

24. Tajarobi F, Abrahmsén-Alami S, Carlsson AS, Larsson A. Simultaneous probing of swelling, erosion and dissolution by NMR-microimaging-Effect of solubility of additives on HPMC matrix tablets. Eur J Pharm Sci. 2009;37(2):89-97.

25. Siepmann J, Peppas NA. Modeling of drug release from delivery systems based on hydroxypropyl methylcellulose (HPMC). Adv Drug Deliv Rev Elsevier BV. 2012;64:163-74.

26. Asare-Addo K, Conway BR, Larhrib H, Levina M, RajabiSiahboomi AR, Tetteh J, et al. The effect of $\mathrm{pH}$ and ionic strength of dissolution media on in-vitro release of two model drugs of different solubilities from HPMC matrices. Colloids Surf B Biointerfaces Elsevier BV. 2013;111:384-91.

27. Bajwa GS, Hoebler K, Sammon C, Timmins P, Melia CD. Microstructural imaging of early gel layer formation in HPMC matrices. J Pharm Sci. 2006;95(10):2145-57.

28. Mitchell K, Ford JL, Armstrong DJ, Elliott PNC, Rostron C, Hogan JE. The influence of additives on the cloud point, disintegration and dissolution of hydroxypropylmethylcellulose gels and matrix tablets. Int J Pharm. 1990;66(1-3):233-42.

29. Pilšar M, Brelih H, Mrhar A, Bogataj M. Analysis of small intestinal transit and colon arrival times of non-disintegrating tablets administered in the fasted state. Eur J Pharm Sci. 2015;75:131-41.

30. Cassilly D, Kantor S, Knight LC, Maurer AH, Fisher RS, Semler J, et al. Gastric emptying of a non-digestible solid: assessment with simultaneous SmartPill $\mathrm{pH}$ and pressure capsule, antroduodenal manometry, gastric emptying scintigraphy. Neurogastroenterol Motil. 2008;20(4):311-9.

31. Radwan A, Amidon GL, Langguth P. Mechanistic investigation of food effect on disintegration and dissolution of BCS class III compound solid formulations: the importance of viscosity. Biopharm Drug Dispos. 2012;33(7):403-16.

32. Schiller C, Fröhlich CP, Giessmann T, Siegmund W, Mönnikes H, Hosten $\mathrm{N}$, et al. Intestinal fluid volumes and transit of dosage forms as assessed by magnetic resonance imaging. Aliment Pharmacol Ther. 2005;22(10):971-9.

33. Weitschies W, Wedemeyer RS, Kosch O, Fach K, Nagel S, Söderlind E, et al. Impact of the intragastric location of extended release tablets on food interactions. J Control Release. 2005;108(23):375-85.

34. Karlsson MO, Sheiner LB. The importance of modeling interoccasion variability in population pharmacokinetic analyses. J Pharmacokinet Biopharm. 1993;21(6):735-50.

35. Dressman JB, Berardi RR, Dermentzoglou LC, et al. Upper gastrointestinal (GI) pH in young, healthy men and women. Pharm Res. 1990; 7:756-61.

36. Evans DF, Pye G, Bramley R, Clark AG, Dyson TJ, Hardcastle JD. Measurement of gastrointestinal $\mathrm{pH}$ profiles in normal ambulant human subjects. Gut. 1988;29:1035-41.

37. Wan X, Wang W, Liu J, Tong T. Estimating the sample mean and standard deviation from the sample size, median, range and/or interquartile range. BMC Med Res Methodol. 2014;14:135. 